\title{
Time-reversible Hamiltonian vector fields with symplectic symmetries
}

\author{
C.A. Buzzi* M.A. Teixeira ${ }^{\dagger}$
}

April 28, 2004

\begin{abstract}
This paper deals with the dynamics of time-reversible Hamiltonian vector fields with 2 degrees of freedom around an elliptic equilibrium point in presence of $1:-1$ resonance. The main result says that under certain conditions there are 2 one-parameter families of reversible periodic solutions terminating at the equilibrium.
\end{abstract}

\section{Introduction}

In 1895 Liapunov published his celebrated center theorem (see for example in Abraham and Marsden [1] p 498). Generalizations of such result were made by Weinstein [20] and Moser [14]. They concern on the existence of $n$ families of periodic solutions filling up smooth 2-dimensional manifolds going through an elliptic equilibrium point. Other generalized versions of such result came up: Devaney [7] considered time-reversible systems, Golubitisky-Krupa-Lim [8] and Montaldi-Roberts-Stewart [13] studied classes of equivariant systems.

Let $X$ be a (germ of a) smooth vector field on $R^{N}$ with $X(0)=0$. The vector field is called time-reversible if there is a germ of a smooth involution $\phi: R^{N}, 0 \rightarrow R^{N}, 0$ $\left(\phi^{2}=I d\right)$ satisfying the relation

$$
X(\phi(x))=-\phi^{\prime}(x) \cdot X(x) \quad, \quad x \in R^{N}, 0
$$

*Partially supported by Fapesp-Brazil under the grants 02/06531-3 and Procad/Capes-Brazil 0092/01-0 TIPO I.

${ }^{\dagger}$ Partially supported by Fapesp-Brazil under the grant 02/10246-2 and Pronex-Brazil grant 76.97.1080/00. 
The main aim of this paper is to study the existence of one parameter families of periodic orbits passing through a typical singularity for smooth time-reversible Hamiltonian systems with respect to a symplectic involution. Our systems belong to the class of vector fields presenting a $1:-1$ resonance at the equilibrium (in two-degrees of freedom). We observe that only two distinct non-trivial symplectic involutions can arise. Moreover these symplectic involutions force the system to have a non-positive definite Hessian, and the linearized system to be semi-simple 1 : -1 resonant. Examples of such systems were exhibited in [6] and [10].

Our approach consists in using Birkhoff normal form, adapted to time-reversible systems with respect to a symplectic involution, to find a formal normal form which has its second order terms as a first integral. We use this formal normal form and transversality theory, restricted to the energy surfaces, to prove that the periodic solutions of the linearized system persist. In the present paper we start the problem by considering the problem for one degree of freedom. We point out that our main result is given by Theorem B. Roughly speaking, it exhibits conditions on systems of 2 degrees of freedom for which there are two one-parameter family of reversible periodic orbits passing through the equilibrium point.

We first study time-reversible Hamiltonian systems of one degree of freedom. In our setting the problem is carried out to consider planar vector fields that are $(-I d)$ reversible. So we know that such systems have no periodic orbits that encircle the equilibrium point. We present an anti-invariant version of a result of Poènaru [15] for mappings on $\mathbb{R}^{n}$. This result will be very useful throughout the paper. We also apply such result to derive the classification of generic two-parameter families of reversible Hamiltonian vector fields of one degree of freedom.

This paper is organized as follows. In section 2 we briefly recall basics concepts of time-reversible systems, introduce some notations and definitions and establish the Theorem 1 to be used in the sequel. In section 3.1, we study Hamiltonian systems with one degree of freedom. In section 3.2, we work with time-reversible Hamiltonian systems with two degrees of freedom.

Acknowledgments. We wish to express our gratitude to the referee for the improvement of the Theorem B and many others helpful suggestions.

\section{Preliminaries}

\subsection{Reversible Systems}

In this section we recall some basic definitions, concepts and results concerning reversible vector fields.

A $C^{k}$ diffeomorphism $\varphi: \mathbb{R}^{n} \rightarrow \mathbb{R}^{n}$ is called an involution if $\varphi \circ \varphi=I d$. 
Definition 1 Given an involution $\varphi: \mathbb{R}^{n} \rightarrow \mathbb{R}^{n}$ we say that a $C^{k}$ vector field $X$ over $\mathbb{R}^{n}$ is $\varphi$-reversible, if $\varphi_{*} X=-X \circ \varphi$, i. e., $d \varphi_{p}(X(p))=-X(\varphi(p))$.

Let $S$ be the fixed points set of $\varphi$. An orbit $\gamma$ is said symmetric if $\varphi(\gamma)=\gamma$. Hence, every critical point of $X$ in $S$ is a symmetric singularity of $X$. Some classical properties of reversible systems are:

- The phase portrait of $X$ is symmetric with respect to $S$.

- A symmetric critical point or symmetric periodic orbit can not be attractor or repellor.

- If $X(p)=0$ and $p \notin S$ then $X(\varphi(p))=0$.

- If a regular orbit $\gamma$ intersects $S$ in two distinct points then $\gamma$ is a periodic orbit.

- If $X(p) \neq 0$ and $p \in S$ then $X(p) \notin T_{p} S$.

- Any periodic orbit $\gamma$ of $X$ not crossing $S$ is paired by another periodic solution given by $\varphi(\gamma)$.

The concept of structural stability comes from the following definition:

Definition 2 Two vector fields $X_{1}$ and $X_{2}$ are said $C^{k}$-orbitally equivalent, $k \geq 1$, if there is a $C^{k}$-diffeomorphism $h$ in the phase space that sends trajectories of $X_{1}$ in trajectories of $X_{2}$ preserving the orientation, but not necessarily the time. If $h$ preserves the time, $i$. e., dh० $X_{1}=X_{2} \circ h$, then $X_{1}$ and $X_{2}$ are $C^{k}$-conjugated.

Definition 3 Given a vector field $X_{0}$, a family $\left(X_{\lambda}\right)_{\lambda \in \Lambda}$ is an unfolding to $X_{0}$ if there exists $\lambda_{0} \in \Lambda$ such that $X_{\lambda_{0}}=X_{0}$. An unfolding $\left(X_{\mu}\right)$ is $C^{k}$-versal, $k \geq 1$, if given another unfolding $\left(Y_{\lambda}\right)$ of $X_{\mu_{0}}$ there is a $C^{k}$-reparametrization $\lambda \mapsto \mu(\lambda)$ such that for all parameters $\lambda$, the systems $Y_{\lambda}$ and $X_{\mu(\lambda)}$ are $C^{k}$-orbitally equivalent.

In the same way, we can define unfoldings and versal unfoldings for maps using the concept of right-equivalence to be aborded in the next section.

\subsection{Hamiltonian Vector Fields}

We consider (germs of) smooth functions $H: \mathbb{R}^{2 n}, 0 \rightarrow \mathbb{R}$ having the origin as a critical point. The corresponding Hamiltonian vector field, to be denoted by $X_{H}$, has the origin as an equilibrium or singular point. We here recall that $d H=\omega\left(X_{H},.\right)$, where $\omega=d x_{1} \wedge d y_{1}+d x_{2} \wedge d y_{2}+\cdots+d x_{n} \wedge d y_{n}$ denotes the standard 2-form on $\mathbb{R}^{2 n}$. In coordinates $X_{H}$ is expressed as:

$$
\dot{x}_{i}=\frac{\partial H}{\partial y_{i}}, \quad \dot{y}_{i}=-\frac{\partial H}{\partial x_{i}} \text { for } i=1,2, \ldots, n .
$$


Definition 4 Let $H$ and $K$ be two functions defined in $V$. We say that $H$ is $C^{k}$-right equivalent to $K$ if there exists a $C^{k}$ diffeomorphism $\Phi: V \rightarrow V$ such that $H=K \circ \Phi$.

We will see that, in dimension two, if $H$ and $K$ are $C^{k}$-right equivalent then the Hamiltonian vector fields $X_{H}$ and $X_{K}$ are $C^{k}$-orbitally equivalent. In dimension greater than two, $H$ and $K$ being $C^{k}$-right equivalent does not imply that $X_{H}$ and $X_{K}$ are $C^{k}$ - orbitally equivalent. But if $\Phi$ is a $C^{k}$-right equivalence between $H$ and $K$ and preserves the symplectic structure then it is a $C^{k}$-conjugacy between $X_{H}$ and $X_{K}$.

Let's define a vector field induced by a diffeomorphism $\Phi$.

Definition 5 Let $X_{H}$ be a Hamiltonian vector field and $\Phi$ be a $C^{k}$-diffeomorphism. We say that the vector field $\Phi_{*}\left(X_{H}\right)(\Phi(p)):=d \Phi_{p}\left(X_{H}(p)\right)$ is induced from $X_{H}$ by $\Phi$.

We observe that $X_{H}$ and $\Phi_{*}\left(X_{H}\right)$ are always $C^{k}$-conjugate.

Lemma 1 [3] Let $H$ and $K$ be functions defined in an open subset of $\mathbb{R}^{2}$. Suppose that there is a $C^{k}$-diffeomorphism $\Phi$ such that $H=K \circ \Phi$. If $X_{H}$ and $X_{K}$ are Hamiltonian vector fields, with respect to $H$ and $K$ respectively, then $\Phi_{*}\left(X_{H}\right)=$ $($ det $d \Phi) X_{K}$. Consequently $X_{H}$ and $X_{K}$ are $C^{k}$-orbitally equivalent.

Proof We transport the equation $d H=\omega\left(X_{H},.\right)$ by $\Phi$. Write $\Phi_{*}(H):=H \circ$ $\Phi^{-1}=K$ and $\Phi_{*}(\omega)=\left(\Phi^{-1}\right)^{*}(\omega)$, so $d K=d \Phi_{*}(H)=\Phi_{*}(\omega)\left(\Phi_{*} X_{H},.\right)$. Here $\Phi_{*}(\omega)=(\operatorname{det} d \Phi)^{-1} \omega$, hence $d K=(\operatorname{det} d \Phi)^{-1} \omega\left(\Phi_{*}\left(X_{H}\right),.\right)$, therefore we have that $d K=\omega\left((\operatorname{det} d \Phi)^{-1} \Phi_{*}\left(X_{H}\right),.\right)$ and $(\operatorname{det} d \Phi)^{-1} \Phi_{*}\left(X_{H}\right)=X_{K}$. Since the vector fields $X_{H}$ and $\Phi_{*}\left(X_{H}\right)$ are $C^{k}$-conjugate, the presence of the scalar factor det $d \Phi$ implies that the Hamiltonian vector fields $X_{H}$ and $X_{K}$ are $C^{k}$-orbitally equivalent, which immediately proves the lemma.

Remark 1 We observe here that the main step in the above proof is the relation $\Phi_{*}(\omega)=(\text { det } d \Phi)^{-1} \omega$. This is true only in dimension two. In dimension greater than two, we must suppose that $\Phi$ preserves the symplectic structure, in the sense of the next definition.

Definition 6 A diffeomorphism $\Phi$ is symplectic if $\Phi^{*}(\omega)=\omega, i$. e., $\omega\left(v_{p}, w_{p}\right)=$ $\omega\left(d \Phi_{p}\left(v_{p}\right), d \Phi_{p}\left(w_{p}\right)\right)$.

Proposition 1 If $\Phi$ is symplectic and is a $C^{k}$-right equivalence between $H$ and $K$, then it is a $C^{k}$-conjugacy between $X_{H}$ and $X_{K}$.

The proof of this proposition is practically the same as lemma 1, considering the remark 1.

The next proposition gives a normal form for symplectic involutions. 
Proposition 2 Fixed the symplectic structure $\omega$ and given an involution $\varphi$, there exists a symplectic change of coordinates that transform $\varphi$ into the normal form

$$
\varphi_{0}\left(x_{1}, y_{1}, x_{2}, y_{2}\right)=\left(x_{1}, y_{1},-x_{2},-y_{2}\right) \text { or } \varphi_{0}=I d \text { or } \varphi_{0}=-I d \text {. }
$$

Before the proof we observe that the mapping $\sigma=\frac{1}{2}(\varphi+d \varphi)$ is a symplectic conjugacy between $\varphi$ and $d \varphi$, i. e., $\varphi \circ \sigma=\sigma \circ d \varphi$. This means that we can assume $\varphi$ is linear.

Lemma 2 If $\varphi$ is a linear symplectic involution, then $\mathbb{R}^{4}=F i x(\varphi) \oplus$ Fix $(-\varphi)$ and $\omega(\operatorname{Fix}(\varphi), \operatorname{Fix}(-\varphi))=0$.

Proof For every $u \in \mathbb{R}^{4}$, we can write $u=\frac{u+\varphi(u)}{2}+\frac{u-\varphi(u)}{2}$. Observe that $\frac{u+\varphi(u)}{2} \in$ $F i x(\varphi)$ and $\frac{u-\varphi(u)}{2} \in F i x(-\varphi)$. Let $u$ be in Fix $(\varphi)$ and $v$ be in Fix $(-\varphi)$, so we have that $\omega(u, v)=\omega(\varphi(u),-\varphi(v))$. By using that $\varphi$ is symplectic, i.e., $\omega(\varphi(u), \varphi(v))=$ $\omega(u, v)$ we have that $\omega(u, v)=-\omega(u, v)=0$. So $\omega(\operatorname{Fix}(\varphi), F i x(-\varphi))=0$.

A linear subspace $U$ of $\mathbb{R}^{4}$ is symplectic if $\omega$ is non-degenerate in $U$, i.e., if $\omega(u, v)=$ 0 for all $u \in U$ then $v=0$.

Lemma 3 Fix $(\varphi)$ and Fix $(-\varphi)$ are symplectic subspaces.

Proof Suppose $u \in F i x(\varphi)$ and $u \neq 0$ such that $\omega(u, F i x(\varphi))=0$. By using lemma 2, we have $\omega(F i x(\varphi), F i x(-\varphi))=0$, so $\omega(u, F i x(-\varphi))=0$. Again by lemma $2\left(\mathbb{R}^{4}=F i x(\varphi) \oplus \operatorname{Fix}(-\varphi)\right)$ we have $\omega\left(u, \mathbb{R}^{4}\right)=0$ and so $\omega$ is degenerate in $\mathbb{R}^{4}$ which is not true. So Fix $(\varphi)$ is a symplectic subspace. The proof for Fix $(-\varphi)$ is analogous.

Proof of proposition 2 By using lemmas 2 and 3 , if $\varphi_{0} \neq I d$ and $\varphi_{0} \neq-I d$, we can find symplectic basis $\left\{e_{1}, f_{1}\right\}$ for Fix $(\varphi)$ and $\left\{e_{2}, f_{2}\right\}$ for Fix $(-\varphi)$ such that $\omega\left(e_{i}, f_{j}\right)$ is equal to 1 if $i=j$ and 0 if $i \neq j$, and $\omega\left(e_{i}, e_{j}\right)=\omega\left(f_{i}, f_{j}\right)=0$ for all $i$ and $j$. Note that $\varphi\left(e_{i}\right)$ and $\varphi\left(f_{i}\right)$ are equal to 1 if $i=1$ and -1 if $i=2$. So in these coordinates $\varphi$ has the normal form given in proposition.

\subsection{The Anti-invariant Theory}

Hamßmann, in [9], studied Hamiltonian vector fields $X_{H}$, where $H$ is $G$-invariant, i. e., $H(g x)=H(x)$ for all $g \in G$. We can not apply this theory here, because we will see in proposition 3 that our $H$ satisfies $H(\varphi x)=-H(x)$. Observe that if $H\left(g_{1} x\right)=-H(x)$ and $H\left(g_{2} x\right)=-H(x)$, then $H\left(g_{1} g_{2} x\right)=H(x)$. So, we decided to work with $G_{\varphi}$-anti-invariant functions to be defined in the following way. 
Definition 7 Let $\varphi$ be a linear involution in $\mathbb{R}^{n}$ and $G$ be a compact group acting linearly on $\mathbb{R}^{n}$. If $f \in \mathcal{H}$ we say that $f$ is $G_{\varphi}$-anti-invariant if:

$$
\begin{aligned}
& f(g x)=f(x) \forall g \in G \text { and } \\
& f(\varphi x)=-f(x)
\end{aligned}
$$

where $\mathcal{H}$ denotes the set of all (germs of) smooth functions.

The notation to the set of (germs of) $G_{\varphi}$-anti-invariant functions will be $\mathcal{H}^{G}$. If $G=\{I d\}$ and $f$ is $G_{\varphi}$-anti-invariant we simply say that $f$ is $\varphi$-anti-invariant.

Definition 8 A vector field $X(x)$ will be said $G_{\varphi}$-equivariant if $X(\varphi x)=\varphi X(x)$ and $X(g x)=T g(X(x))=g X(x)$ for all $g \in G$. We denote by $\mathscr{X}^{G}$ the set of all (germs of) $G_{\varphi}$-equivariant vector fields.

Lemma 4 If $f \in \mathcal{H}^{G}$ then

$$
\begin{aligned}
& <d f(g x), v>=<d f(x), g^{-1} v>\forall g \in G, \forall v \in \mathbb{R}^{n} \text { and } \\
& <d f(\varphi x), v>=-<d f(x), \varphi v>\forall v \in \mathbb{R}^{n} .
\end{aligned}
$$

where $<., .>$ is the standard inner product on $\mathbb{R}^{n}$.

Proof Fix $g \in G$ and let $L$ be its left multiplying, i. e., $L: x \mapsto g x$. In this case we have $f \circ L=f$, so $d f_{x}=d f_{L(x)} \circ d L_{x}$. Therefore $d f_{x}(w)=d f_{g x}(g w)$ for all $w \in \mathbb{R}^{n}$. In another words, $\langle d f(x), w\rangle=\left\langle d f(g x), g w>\right.$, so $\left.<d f(x), g^{-1} w\right\rangle=$ $<d f(g x), w>$ for all $w \in \mathbb{R}^{n}$. In the second case we get analogous result with the minus sign and using $d f_{x}=-d f_{\varphi(x)} \circ d \varphi_{x}$.

Lemma 5 If $f \in \mathcal{H}^{G}$ and $X \in \mathfrak{X}^{G}$ then $d f(X) \in \mathcal{H}^{G}$.

Proof By using the previous lemma, fix $g \in G$, so $d f(X)(g x)=<d f(g x), X(g x)>$ $=<d f(g x), g X(x)>=<d f(x), g^{-1} g X(x)>=<d f(x), X(x)>=d f(X)(x)$. And $d f(X)(\varphi x)=<d f(\varphi x), X(\varphi x)>=<d f(\varphi x), \varphi X(x)>=-<d f(x), \varphi^{2} X(x)>=$ $-<d f(x), X(x)>=-d f(X)(x)$.

Now we define the $G_{\varphi}$-anti-invariant Jacobian ideal.

Definition 9 The $G_{\varphi}$-anti-invariant Jacobian ideal is defined by

$$
\mathcal{J}_{G}(f)=\left\{d f(X): X \in \mathfrak{X}^{G}\right\} .
$$


Let $d \mu(g)$ be a Haar measure in $G$ such that $\int_{G} d \mu(g)=1$, and let's define the following evaluation maps $A v: \mathcal{H} \longrightarrow \mathcal{H}^{G}$ by

$$
[A v(f)](x)=\frac{1}{2} \int_{G}[f(g x)-f(\varphi g x)] d \mu(g)
$$

and $A v: \mathfrak{X} \longrightarrow \mathfrak{X}^{G}$ by

$$
[A v(X)](x)=\frac{1}{2} \int_{G}\left[g^{-1} X(g x)+g^{-1} \varphi X(\varphi g x)\right] d \mu(g) .
$$

The next theorem is a $G_{\varphi}$-anti-invariant version of a result due to Poènaru [15] and it is the main result of this section.

Theorem 1 Let $f(x) \in \mathcal{H}^{G}$ be such that $\operatorname{dim}_{\mathbb{R}} \mathcal{H} / \mathcal{J}(f)<\infty$. If the functions $\varphi_{1}, \ldots, \varphi_{k}$ are a basis for $\mathcal{H} / \mathcal{J}(f)$ then $A v\left(\varphi_{1}\right), \ldots, A v\left(\varphi_{k}\right)$ span the real vector space $\mathcal{H}^{G} / \mathcal{J}_{G}(f)$.

Proof Let $h \in \mathcal{H} / \mathcal{J}(f)$. There are $\lambda_{1}, \ldots, \lambda_{k} \in \mathbb{R}$ and $X \in \mathfrak{X}$ such that

$$
h(x)=\sum_{i=1}^{k} \lambda_{i} \varphi_{i}(x)+<d f(x), X(x)>.
$$

By hypothesis $f \in \mathcal{H}^{G}$, using the lemma 4 we have

$$
\begin{gathered}
A v(<d f(x), X(x)>)=\frac{1}{2} \int_{G}[<d f(g x), X(g x)>-<d f(\varphi g x), X(\varphi g x)>] d \mu(g)= \\
=\frac{1}{2} \int_{G}\left[<d f(x), g^{-1} X(g x)>+<d f(g x), \varphi X(\varphi g x)>\right] d \mu(g)= \\
=\frac{1}{2} \int_{G}\left[<d f(x), g^{-1} X(g x)>+<d f(x), g^{-1} \varphi X(\varphi g x)>\right] d \mu(g)= \\
=<d f(x), \frac{1}{2} \int_{G}\left[g^{-1} X(g x)+g^{-1} \varphi X(\varphi g x)\right] d \mu(g)>= \\
=<d f(x),[A v(X)](x)>.
\end{gathered}
$$

Therefore, considering $h(x) \in \mathcal{H}^{G}$, we have that

$$
h(x)=A v(h(x))=\sum_{i=1}^{k} \lambda_{i} A v\left(\varphi_{i}\right)+<d f, A v(X)>,
$$

and so, we have that $A v\left(\varphi_{1}\right), \ldots, A v\left(\varphi_{k}\right)$ span $\mathcal{H}^{G} / \mathcal{J}_{G}(f)$. 
The relationship between the $\varphi$-reversible vector field and the Hamiltonian function is given by the next proposition.

Proposition 3 Let $\varphi$ be a symplectic involution. A $C^{k}$ Hamiltonian vector field $X_{H}$ is $\varphi$-reversible if, and only if, the Hamiltonian function $H$ is $\varphi$-anti-invariant.

Proof $X_{H}$ is $\varphi$-reversible iff $X_{H}(\varphi(x))=-\varphi \circ X_{H}(x)$ for all $x \in \mathbb{R}^{2 n}$, i. e., $\omega\left(X_{H}(\varphi(x)), \varphi(y)\right)=-\omega\left(\varphi X_{H}(x), \varphi(y)\right)$ for all $x, y \in \mathbb{R}^{2 n}$. By hypothesis, $\varphi$ is symplectic. Thus $\omega\left(X_{H}(\varphi(x)), \varphi(y)\right)=-\omega\left(X_{H}(x), y\right)$ for all $x, y \in \mathbb{R}^{2 n}$, i. e., $-d H_{x}(y)=d H_{\varphi(x)}(\varphi(y))=d(H \circ \varphi)_{x}(y)$ for all $x, y \in \mathbb{R}^{2 n}$. It is equivalent to $H \circ \varphi=-H$.

\section{$3 \quad$ Hamiltonian reversible vector fields}

The symplectic involutions in $\mathbb{R}^{2}, 0$ are $I d$ and $-I d$. The symplectic involutions in $\mathbb{R}^{4}, 0$, according to proposition 2 , are $I d,-I d$ or $\varphi_{1}\left(x_{1}, y_{1}, x_{2}, y_{2}\right)=\left(x_{1}, y_{1},-x_{2},-y_{2}\right)$.

We observe that if a vector field $X$ is reversible with respect to $I d$ then $X=0$. So we will consider only the cases where the involution is not the $I d$.

\subsection{Hamiltonian with one-degree of freedom}

First of all we recall that in [9] Hamßmann classified the generic codimension two singularities and presented the respective versal unfoldings of Hamiltonian reversible vector fields with respect to an anti-symplectic involution. In [5] there is a general classification of planar singularities of $(-I d)$-reversible vector fields. Here we classify generically the codimension two symmetric singularities by presenting their versal unfoldings in the case of symplectic time-reversing symmetry.

Due to an orientation problem, if the vector field is reversible with respect to $-I d$ on the plane, then there is no periodic solution that encircles the origin. So, there is no Liapunov center families in one degree of freedom. On the other hand, using the anti-invariant theory described in section 2.3 we can derive the behavior of the dynamics near a symmetric equilibrium point by showing their versal unfoldings.

If $X_{H}$ is $(-I d)$-reversible, then $H$ belongs to $\mathcal{H}^{G}$ and the origin is a critical point of $H$, so its second jet at 0 is null. The possible non-vanishing terms are those of order three. We know, from singularity theory, (see [2]) that the generic functions with zero 2-jet have the following normal forms $f^{1}(x, y)=x^{3}+x y^{2}$ and $f^{2}(x, y)=x^{3}-x y^{2}$. Their versal unfoldings, in the world of all smooth functions, are

$$
f_{\lambda, \mu, \eta}^{1}(x, y)=x^{3}+x y^{2}+\lambda\left(x^{2}-y^{2}\right)+\mu x+\eta y
$$

and

$$
f_{\lambda, \mu, \eta}^{2}(x, y)=x^{3}-x y^{2}+\lambda\left(x^{2}+y^{2}\right)+\mu x+\eta y,
$$


respectively.

\section{Theorem A}

(i) If $X_{H}: \mathbb{R}^{2}, 0 \rightarrow \mathbb{R}^{2}$ is $(-I d)$-reversible, then there is no Liapunov center families for $X_{H}$.

(ii) The versal unfoldings of $f^{1}(x, y)=x^{3}+x y^{2}$ and $f^{2}(x, y)=x^{3}-x y^{2}$ in $\mathcal{H}^{G}$ are $f_{\mu, \eta}^{1}(x, y)=x^{3}+x y^{2}+\mu x+\eta y$ and $f_{\mu, \eta}^{2}(x, y)=x^{3}-x y^{2}+\mu x+\eta y$, respectively.

(iii) The two-parameter family of reversible Hamiltonian vector fields

$$
X_{\mu, \eta}^{1}:\left\{\begin{array}{l}
\dot{x}=2 x y+\eta \\
\dot{y}=-3 x^{2}-y^{2}-\mu
\end{array} \quad \text { and } \quad X_{\mu, \eta}^{2}:\left\{\begin{array}{l}
\dot{x}=2 x y+\eta \\
\dot{y}=-3 x^{2}+y^{2}-\mu
\end{array}\right.\right.
$$

are the $C^{\infty}$-versal unfoldings of $X_{0,0}^{1}$ and $X_{0,0}^{2}$ respectively.

Proof (i) Any vector field on the plane that is $(-I d)$-reversible cannot have periodic solutions that encircle the origin.

(ii) It is a immediate application of theorem 1 to the previous versal unfoldings.

(iii) Given an unfolding $X_{K_{\lambda}}$ of $X_{0,0}^{1}$, we have that $K_{\lambda}$ is also an unfolding of $f^{1}$. By (ii), $f_{\mu, \eta}^{1}$ is a $C^{\infty}$-versal unfolding of $f^{1}$. So there exists a $C^{\infty}$-reparametrization $\lambda \mapsto(\mu(\lambda), \eta(\lambda))$ such that for all $\lambda, K_{\lambda}$ and $f_{\mu(\lambda), \eta(\lambda)}^{1}$ are $C^{\infty}$-right equivalent. From lemma 1 we deduce that $X_{K_{\lambda}}$ and $X_{\mu(\lambda), \eta(\lambda)}^{1}$ are $C^{\infty}$-equivalent.

The figures 1 and 2 show the bifurcation diagram of these families.

\subsection{Hamiltonian with two-degrees of freedom}

If $X_{H}$ is a $(-I d)$-reversible Hamiltonian vector field then, from proposition 3 , the Hamiltonian function $H$ has only monomials of odd order. So the linear part of $X_{H}$ is zero. This case is very degenerate and its analysis is addressed to a forthcoming paper.

Throughout this section we are assuming that $X_{H}$ is $\varphi_{1}$-reversible, where $\varphi_{1}$ is given by $\varphi_{1}\left(x_{1}, y_{1}, x_{2}, y_{2}\right)=\left(x_{1}, y_{1},-x_{2},-y_{2}\right)$. From proposition 3 , the function $H$ satisfies $H \circ \varphi=-H$. So, the second order terms of $H$ are

$$
H_{2}=a x_{1} x_{2}+b x_{1} y_{2}+c y_{1} x_{2}+d y_{1} y_{2}
$$

If $b c-a d>0$, then the eigenvalues of the linear part of $X_{H}$ are $\{\beta, \beta,-\beta,-\beta\}$, where $\beta=\sqrt{b c-a d}$. We are interested in elliptic equilibria, so this case will not be treated here.

If $b c-a d<0$, then the eigenvalues of the linear part of the vector field $X_{H}$ are $\{\alpha i, \alpha i,-\alpha i,-\alpha i\}$, where $\alpha=\sqrt{a d-b c}$. We will conclude that this is a semi-simple $1:-1$ resonant case. 


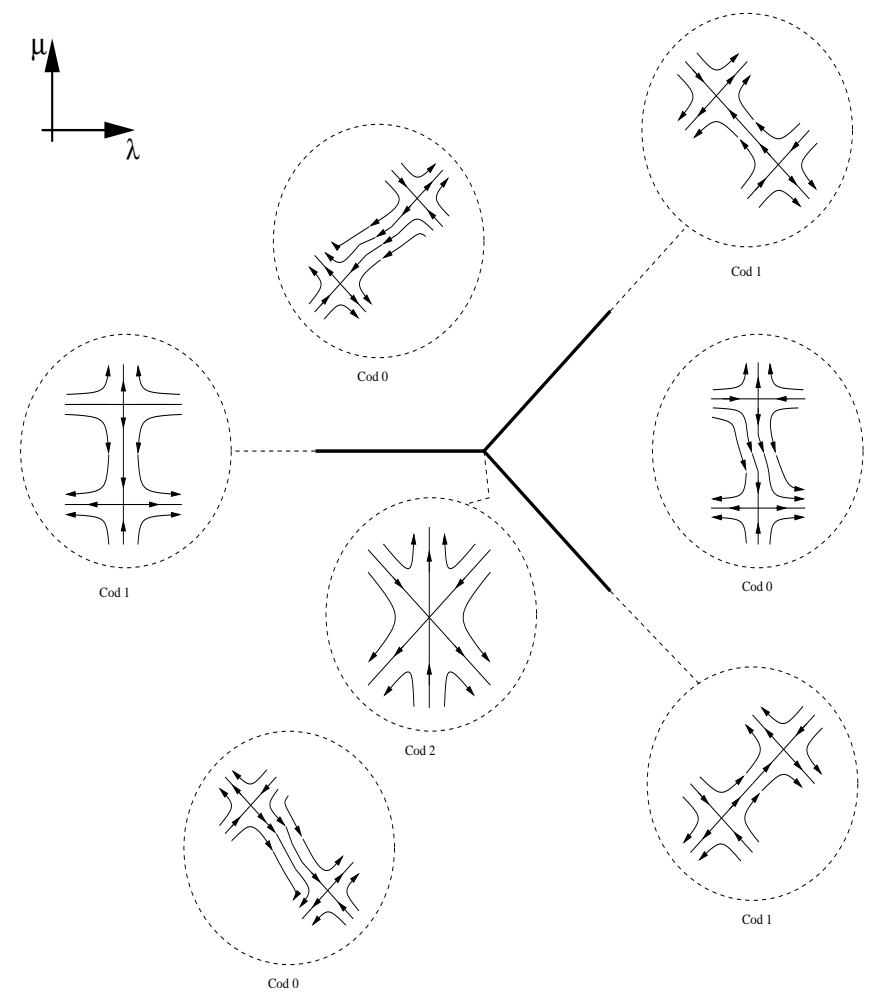

Figure 1: Bifurcation diagram of $X_{\lambda, \mu}^{2}$

By means of the symplectic change of coordinates

$$
M:\left\{\left[\begin{array}{l}
x_{1} \\
y_{1} \\
x_{2} \\
y_{2}
\end{array}\right]=\left[\begin{array}{cccc}
-c / \alpha & -d / \alpha & 0 & 0 \\
a / \alpha & b / \alpha & 0 & 0 \\
0 & 0 & 1 & 0 \\
0 & 0 & 0 & 1
\end{array}\right] \cdot\left[\begin{array}{c}
\widetilde{x_{1}} \\
\widetilde{y_{1}} \\
\widetilde{x_{2}} \\
\widetilde{y_{2}}
\end{array}\right]\right.
$$

we obtain

$$
\begin{aligned}
H_{2}\left(\widetilde{x_{1}}, \widetilde{y_{1}}, \widetilde{x_{2}}, \widetilde{y_{2}}\right) & =\alpha\left(\widetilde{x_{1}} \widetilde{y_{2}}-\widetilde{y_{1}} \widetilde{x_{2}}\right) \\
\varphi_{1}\left(\widetilde{x_{1}}, \widetilde{y_{1}}, \widetilde{x_{2}}, \widetilde{y_{2}}\right) & =\left(\widetilde{x_{1}}, \widetilde{y_{1}},-\widetilde{x_{2}},-\widetilde{y_{2}}\right) \\
X_{H_{2}}\left(\widetilde{x_{1}}, \widetilde{y_{1}}, \widetilde{x_{2}}, \widetilde{y_{2}}\right) & =\left(-\alpha \widetilde{x_{2}},-\alpha \widetilde{y_{2}}, \alpha \widetilde{x_{1}}, \alpha \widetilde{y_{1}}\right) .
\end{aligned}
$$

Observe that the Hessian of $H$ is non-positive definite. Consequently, we cannot apply the results of Weinstein [20] and Moser [14].

Consider

$$
T:\left\{\left[\begin{array}{c}
\widetilde{x_{1}} \\
\widetilde{y_{1}} \\
\widetilde{x_{2}} \\
\widetilde{y_{2}}
\end{array}\right]=\left[\begin{array}{cccc}
\frac{\sqrt{2}}{2} & 0 & \frac{\sqrt{2}}{2} & 0 \\
0 & \frac{\sqrt{2}}{2} & 0 & \frac{\sqrt{2}}{2} \\
-i \frac{\sqrt{2}}{2} & 0 & i \frac{\sqrt{2}}{2} & 0 \\
0 & i \frac{\sqrt{2}}{2} & 0 & -i \frac{\sqrt{2}}{2}
\end{array}\right] \cdot\left[\begin{array}{c}
z_{1} \\
w_{1} \\
z_{2} \\
w_{2}
\end{array}\right]\right.
$$




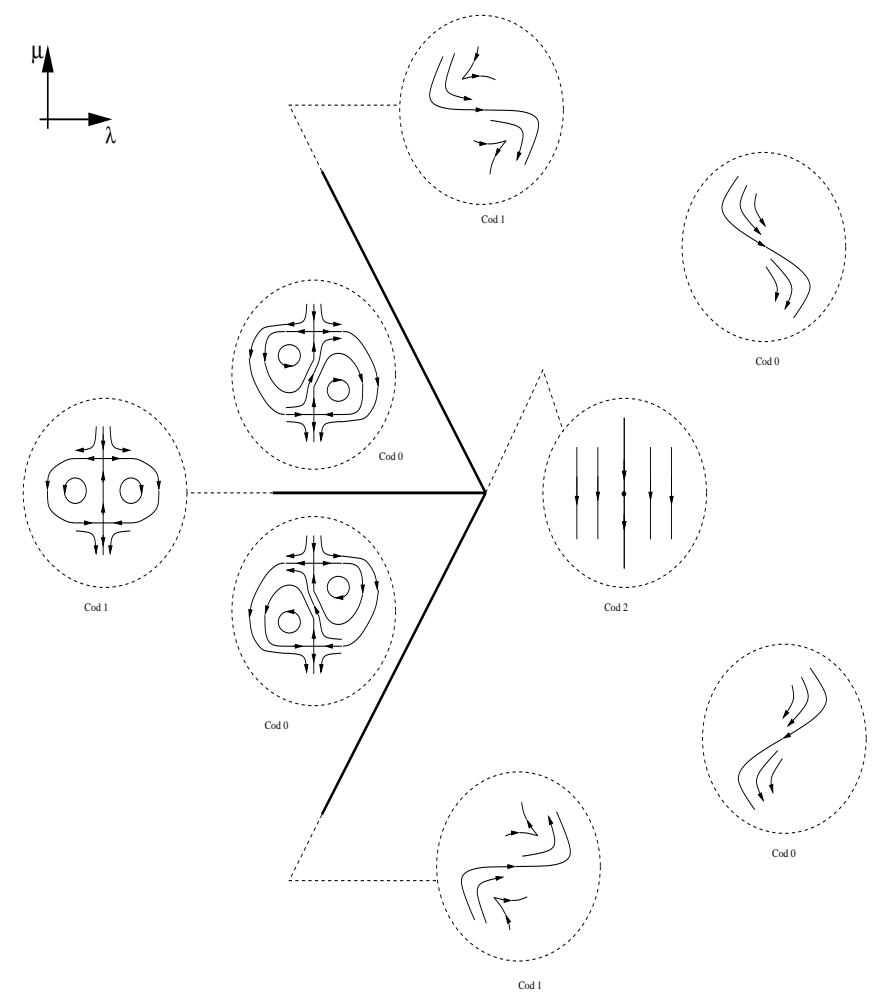

Figure 2: Bifurcation diagram of $X_{\lambda, \mu}^{1}$

This mapping is symplectic and in these new coordinates we have

$$
\begin{aligned}
H_{2}\left(z_{1}, w_{1}, z_{2}, w_{2}\right) & =\alpha i\left(z_{1} w_{1}-z_{2} w_{2}\right) \\
\varphi_{1}\left(z_{1}, w_{1}, z_{2}, w_{2}\right) & =\left(z_{2}, w_{2}, z_{1}, w_{1}\right) \\
X_{H_{2}}\left(z_{1}, w_{1}, z_{2}, w_{2}\right) & =\left(\alpha i z_{1},-\alpha i w_{1},-\alpha i z_{2}, \alpha i w_{2}\right)
\end{aligned}
$$

We observe that, according to Van der Meer [18], this is the semi-simple 1: -1 resonant case.

The function $\{f, g\}=\omega\left(X_{f}, X_{g}\right)$ is called the Poisson bracket of the smooth functions $f$ and $g$. Let $\mathcal{H}_{n}$ be the set of all homogeneous polynomials of degree $n$. The application adjoint $A d_{H_{2}}: \mathcal{H}_{n} \rightarrow \mathcal{H}_{n}$ is defined by

$$
A d_{H_{2}}(H)=\left\{H_{2}, H\right\}=\omega\left(X_{H_{2}}, X_{H}\right)=<-X_{H_{2}}, \nabla H>.
$$

The Birkhoff Normal Form Theorem [16] states that if we have a Hamiltonian $H=H_{2}+H_{3}+H_{4}+\cdots$, where $H_{i} \in \mathcal{H}_{i}$ is the homogeneous part of degree $i$, and $\mathcal{G}_{i} \subset \mathcal{H}_{i}$ satisfies $\mathcal{G}_{i} \oplus \operatorname{Range}\left(A d_{H_{2}}\right)=\mathcal{H}_{i}$, then there exists a formal symplectic power series transformation $\Phi$ such that $H \circ \Phi=H_{2}+\widetilde{H_{3}}+\widetilde{H_{4}}+\cdots$ where $\widetilde{H_{i}} \in$ $\mathcal{G}_{i}(i=3,4 \ldots)$. In particular, if $A d_{H_{2}}$ is semi-simple, as in our case, then $\operatorname{Ker}\left(A d_{H_{2}}\right)$ complements Range $\left(\mathrm{Ad}_{\mathrm{H}_{2}}\right)$. 
As $\varphi_{1}$ is symplectic, the change of coordinates $\Phi$ can be chosen in such a way that $H \circ \Phi$ is $\varphi_{1}$-anti-invariant. In order to see this, we can split $\mathcal{H}_{i}=\mathcal{H}_{i}^{+} \oplus \mathcal{H}_{i}^{-}$, where $\mathcal{H}_{i}^{+}=\left\{H \in \mathcal{H}_{i}: H \circ \varphi_{1}=H\right\}$ and $\mathcal{H}_{i}^{-}=\left\{H \in \mathcal{H}_{i}: H \circ \varphi_{1}=-H\right\}$. If $\varphi_{1}$ is symplectic, then $A d_{H_{2}}\left(\mathcal{H}_{i}^{ \pm}\right)=\mathcal{H}_{i}^{\mp}$. In this case, if $\mathcal{H}_{i}=\mathcal{G}_{i} \oplus A d_{H_{2}}\left(\mathcal{H}_{i}\right)$, then $\mathcal{H}_{i}^{-}=\left(\mathcal{G}_{i} \cap \mathcal{H}_{i}^{-}\right) \oplus A d_{H_{2}}\left(\mathcal{H}_{i}^{+}\right)$. Now we can perform the change of coordinates restricted to $\mathcal{H}_{i}^{-}$.

Let's apply this theorem in our case. For $H=z_{1}^{\alpha_{1}} w_{1}^{\beta_{1}} z_{2}^{\alpha_{2}} w_{2}^{\beta_{2}}$, we have $A d_{H_{2}}(H)=$ $\alpha i\left(\beta_{1}-\alpha_{1}+\alpha_{2}-\beta_{2}\right) z_{1}^{\alpha_{1}} w_{1}^{\beta_{1}} z_{2}^{\alpha_{2}} w_{2}^{\beta_{2}}$. Therefore, the monomial $z_{1}^{\alpha_{1}} w_{1}^{\beta_{1}} z_{2}^{\alpha_{2}} w_{2}^{\beta_{2}}$ is in the kernel of $A d_{H_{2}}$ if, and only if, $\beta_{1}-\alpha_{1}+\alpha_{2}-\beta_{2}=0$.

Lemma 6 The function $H$ does not have monomials of odd order in $\operatorname{Ker}\left(\mathrm{Ad}_{\mathrm{H}_{2}}\right)$.

Proof Let $z_{1}^{\alpha_{1}} w_{1}^{\beta_{1}} z_{2}^{\alpha_{2}} w_{2}^{\beta_{2}}$ be a monomial such that $\beta_{1}+\alpha_{1}+\alpha_{2}+\beta_{2}=2 n+1$, then $\beta_{1}-\alpha_{1}+\alpha_{2}-\beta_{2} \neq 0$.

Lemma 7 Let $H=z_{1}^{\alpha_{1}} w_{1}^{\beta_{1}} z_{2}^{\alpha_{2}} w_{2}^{\beta_{2}}$ be in the kernel of $A d_{H_{2}}$ and suppose that we have $\beta_{1}+\alpha_{1}+\alpha_{2}+\beta_{2}=2 n$. In this case, there are positive integer numbers $p, q, r$ and $s$ that satisfy $H=\left(z_{1} w_{1}\right)^{p}\left(z_{1} z_{2}\right)^{q}\left(w_{1} w_{2}\right)^{r}\left(z_{2} w_{2}\right)^{s}$.

Proof The hypothesis $\left(\beta_{1}+\alpha_{2}\right)+\left(\alpha_{1}+\beta_{2}\right)=2 n$ and $\left(\beta_{1}+\alpha_{2}\right)-\left(\alpha_{1}+\beta_{2}\right)=0$, imply that $\beta_{1}+\alpha_{2}=n$ and $\alpha_{1}+\beta_{2}=n$. Therefore

$$
\text { if } \alpha_{1} \geq \alpha_{2} \text { we take }\left\{\begin{array} { l } 
{ s = 0 } \\
{ r = n - \alpha _ { 1 } } \\
{ q = \alpha _ { 2 } } \\
{ p = \alpha _ { 1 } - \alpha _ { 2 } }
\end{array} \text { and if } \alpha _ { 1 } < \alpha _ { 2 } \text { we take } \left\{\begin{array}{l}
p=0 \\
r=n-\alpha_{2} \\
q=\alpha_{1} \\
s=\alpha_{2}-\alpha_{1}
\end{array}\right.\right. \text {. }
$$

Theorem 2 If $H \in \mathcal{H}^{G}$ and $a d-b c>0$, then there is a formal symplectic change of coordinates $\Phi$ such that $\widetilde{H}=H \circ \Phi=I_{1} . f\left(I_{1}^{2}, I_{2}, I_{3}, I_{4}\right)$ where

$$
\begin{aligned}
& I_{1}=\widetilde{x_{2}} \widetilde{y_{1}}-\widetilde{x_{1}} \widetilde{y_{2}} \\
& I_{2}=\widetilde{x_{1}} \widetilde{y_{1}}+\widetilde{x_{2}} \widetilde{y_{2}} \\
& I_{3}={\widetilde{y_{1}}}^{2}+{\widetilde{y_{2}}}^{2} \\
& I_{4}={\widetilde{x_{1}}}^{2}+{\widetilde{x_{2}}}^{2} .
\end{aligned}
$$

Proof We know by Birkhoff normal form theory [16], that there exists a formal symplectic change of coordinates $\Phi$ in such a way that $H \circ \Phi$ has only terms in the kernel of $A d_{H_{2}}$. By using lemmas 6 and 7 , we have that $H \circ \Phi=g\left(z_{1} w_{1}, z_{2} w_{2}, w_{1} w_{2}, z_{1} z_{2}\right)$. Let

$$
\begin{array}{ll}
I_{1}=-i\left(z_{1} w_{1}-z_{2} w_{2}\right) & =\widetilde{x_{2}} \widetilde{y_{1}}-\widetilde{x_{1}} \widetilde{y_{2}} \\
I_{2}=z_{1} w_{1}+z_{2} w_{2} & =\widetilde{x_{1}} \widetilde{y_{1}}+\widetilde{x_{2}} \widetilde{y_{2}} \\
I_{3}=2 w_{1} w_{2} & ={\widetilde{y_{1}}}^{2}+{\widetilde{y_{2}}}^{2} \\
I_{4}=2 z_{1} z_{2} & ={\widetilde{x_{1}}}^{2}+{\widetilde{x_{2}}}^{2} .
\end{array}
$$


So $H \circ \Phi=\widetilde{g}\left(I_{1}, I_{2}, I_{3}, I_{4}\right)$.

On the other hand, $I_{1} \circ \varphi_{1}=-I_{1}, I_{2} \circ \varphi_{1}=I_{2}, I_{3} \circ \varphi_{1}=I_{3}$ and $I_{4} \circ \varphi_{1}=I_{4}$. Hence $H \circ \Phi=I_{1} \cdot f\left(I_{1}^{2}, I_{2}, I_{3}, I_{4}\right)$.

This theorem confirms the result of Meyer [12], where each real homogeneous polynomial of degree $i$, in the normal form, is a first integral of the original linearized system.

The classical theorem due to Liapunov [1] establishes the existence of families of periodic orbits arising from an equilibrium point for real Hamiltonian systems under the following conditions: (i) the $2 n$ eigenvalues $\pm \lambda_{1}, \pm \lambda_{2}, \ldots, \pm \lambda_{n}$ are distinct, (ii) $\lambda_{1}$ is purely imaginary and (iii) $\frac{\lambda_{p}}{\lambda_{1}}$ is non integer for $p=2,3, \ldots, n$ (non-resonance). Each such family of periodic orbits is parameterized by some real parameter $\mu$ such that as $\mu \rightarrow 0$, the periodic orbits tend towards the equilibrium while their periods tend towards $\frac{2 \pi}{\left|i \lambda_{1}\right|}$. In our context the eigenvalues are $\alpha i$ and $-\alpha i$, and so the classical Liapunov's theorem cannot be applied.

The families

$$
\begin{aligned}
& \gamma_{k}(t)=(k \cos (\alpha t), 0, k \sin (\alpha t), 0) \\
& \sigma_{s}(t)=(0, s \cos (\alpha t), 0, s \sin (\alpha t))
\end{aligned}
$$

are two one-parameter families of periodic solutions as in the Liapunov's theorem for the system

$$
X_{H_{2}}\left(\widetilde{x_{1}}, \widetilde{y_{1}}, \widetilde{x_{2}}, \widetilde{y_{2}}\right)=\left(-\alpha \widetilde{x_{2}},-\alpha \widetilde{y_{2}}, \alpha \widetilde{x_{1}}, \alpha \widetilde{y_{1}}\right) .
$$

The planes $\left\{\widetilde{x_{1}}=\widetilde{x_{2}}=0\right\}$ and $\left\{\widetilde{y_{1}}=\widetilde{y_{2}}=0\right\}$ are 2-dimensional manifolds filled up by periodic orbits.

Theorem B Assume $H \in \mathcal{H}^{G}$ and $a d-b c>0$. Then there exists $\widetilde{H}$, formally $\mathcal{C}^{k}$ right equivalent to $H$, such that the vector field $X_{\widetilde{H}}$ has two one-parameter families of symmetric periodic solutions, with period near $\frac{2 \pi}{\alpha}$, as in the Liapunov's Theorem, going through the equilibrium point. Here $\widetilde{H}=H \circ \Phi$, where $\Phi$ is given by Theorem 2 .

We recall that the referee point out that the above result can be improved in the following way. The statement remains true if one drops 'formal' in the conclusions. To archive this improvement one would have to argue along lines of [18] where similarly first a normal form is obtained and then singularity theory is used to match the periodic orbits of the normal form with those of the 'real' system.

Proof Let's recall that $I_{1}$ is a first integral of $H_{2}$ and $\widetilde{H}$, i.e., $\left\{H_{2}, I_{1}\right\}=0$ and $\left\{\widetilde{H}, I_{1}\right\}=0$. We will perform the proof for the family $\gamma_{k}$. Each orbit of the family $\gamma_{k}$, of periodic solutions of $X_{H_{2}}$, intersects $F i x\left(\varphi_{1}\right)=\left\{\tilde{x_{2}}=\tilde{y_{2}}=0\right\}$ in two points $\left\{p_{1}, p_{2}\right\}$. The reversibility implies that $T_{p_{1}}\left(\gamma_{k}\right) \oplus T_{p_{1}}\left(F i x\left(\varphi_{1}\right)\right)$ has dimension 3. The same thing happens to $p_{2}$. In coordinates $\left(\tilde{x}_{1}, \tilde{y}_{1}, \tilde{x}_{2}, \tilde{y}_{2}\right)$ we have that $I_{1}\left(\tilde{x}_{1}, \tilde{y}_{1}, \tilde{x}_{2}, \tilde{y}_{2}\right)=\tilde{x}_{2} \tilde{y}_{1}-\tilde{x}_{1} \tilde{y}_{2}$. This means that the set $\left\{\left(\tilde{x}_{1}, \tilde{y}_{1}, \tilde{x}_{2}, \tilde{y}_{2}\right)\right.$ : 
$\left.I_{1}\left(\tilde{x}_{1}, \tilde{y}_{1}, \tilde{x}_{2}, \tilde{y}_{2}\right)=0\right\}$ is a 3 -dimensional manifold in every open set $U$, such that $0 \notin U$. Let's take two open sets $U_{1}$ and $U_{2}$ such that $p_{1} \in U_{1}, p_{2} \in U_{2}, 0 \notin U_{1} \cup U_{2}$, $\left\{I_{1}=0\right\} \cap U_{1}=S_{1}$ and $\left\{I_{1}=0\right\} \cap U_{2}=S_{2} . \quad S_{1}$ and $S_{2}$ are 3-dimensional manifolds in $\mathbb{R}^{4}$. Consider Fix $\left(\varphi_{1}\right) \cap U_{1}=\Sigma_{1}$ and Fix $\left(\varphi_{1}\right) \cap U_{2}=\Sigma_{2}$. $\quad \Sigma_{1}$ and $\Sigma_{2}$ are 2-dimensional submanifolds such that $\Sigma_{1} \subset S_{1}$ and $\Sigma_{2} \subset S_{2}$, since $\operatorname{Fix}\left(\varphi_{1}\right)=\left\{\tilde{x_{2}}=\tilde{y_{2}}=0\right\} \subset\left\{I_{1}=0\right\}$. We have that $I_{1}\left(\gamma_{k}(t)\right)=0 \forall t$. Now, let's consider the full system $X_{\widetilde{H}}$, where $\widetilde{H}=H_{2}+$ h.o.t. There is an orbit $\gamma_{k}^{\epsilon}$ for $X_{\widetilde{H}}$, near $\gamma_{k}$, such that $I_{1}\left(\gamma_{k}^{\epsilon}(t)\right)=0 \forall t$, since $I_{1}$ is a first integral for $\widetilde{H}$. Then we have the following situation: $\operatorname{dim}\left(\Sigma_{1}\right)=2, \operatorname{dim}\left(S_{1}\right)=3, \Sigma_{1} \subset S_{1}, \gamma_{k} \cap U_{1} \subset S_{1}$ and $\gamma_{k}$ intersects $\Sigma_{1}$ transversally in $S_{1}$. By transversality theory, we have that $\gamma_{k}^{\epsilon} \cap \Sigma_{1}=\left\{p_{1}^{\epsilon}\right\}$. In analogous way, we have that $\gamma_{k}^{\epsilon} \cap \Sigma_{2}=\left\{p_{2}^{\epsilon}\right\}$. By reversibility we derive that if $\gamma_{k}^{\epsilon} \cap \operatorname{Fix}(\varphi)=\left\{p_{1}^{\epsilon}, p_{2}^{\epsilon}\right\}$ then $\gamma_{k}^{\epsilon}$ is a periodic solution for $X_{\widetilde{H}}$. The Theorem of Continuous and Differential Dependence with Respect to Initial Conditions and Parameters tell us that the periods are near $\frac{2 \pi}{\alpha}$. For the family $\sigma_{s}$ the analysis is similar.

\section{References}

[1] Abraham, R. and Marsden, J. E., Foundations of Mechanics, 2nd edn, Benjamin-Cummings, (1978).

[2] Arnold, V. I., Normal forms for functions near degenerate critical points, the Weyl groups $a_{k}, d_{k}$ and $e_{k}$, and Lagrangian singularities., Funct. Anal. Appl., 6, (1972).

[3] Broer, H. W., Chow, S. N., Kim, Y. and Vegter, G., A normally elliptic Hamiltonian bifurcation, Z. Angew. Math. Phys., 44, 389-432, (1993).

[4] Bridges, T. J., Poisson structure of the reversible 1 : 1 resonance, Physica D, 112, 40-49, (1998).

[5] Buzzi, C. A., Generic one-parameter families of reversible vector fields, In: Real and Complex Singularities, (São Carlos, 1998), 202-214, Chapman \& Hall/CRC Res. Notes Math., 412, Chapman \& Hall/CRC, Boca Raton, (2000).

[6] Chow, S. N. and Kim, Y. Bifurcation of periodic orbits for non-positive definite Hamiltonian systems, Aplicable Analysis, 31, 163-199, (1988).

[7] Devaney, R. L., Reversible diffeomorphisms and flows, Trans. Amer. Math. Soc, 218, 89-113, (1976).

[8] Golubitsky, M., Krupa, M. and Lim, C., Time-reversibility and particle sedimentation, SIAM J. Appl. Math., vol 51, n 1, 49-72, (1991). 
[9] Hanßmann, H., The reversible umbilic bifurcation, Physica D, 112, 8194, (1998).

[10] Heinz, F., Dynamik und Stabilitat eines Hamiltonschen Systems, diplomarbeit, (1998).

[11] Meyer, K. R., Generic bifurcations in Hamiltonian systems, In: Dynamical Systems - Warwick 1974, Lec. Notes Math., 468, 62-70, Springer, (1974).

[12] Meyer, K. R., Normal form for Hamiltonian systems, Celestial Mech., 9, 517-522, (1974).

[13] Montaldi, J., Roberts, M. and Stewart, I., Existence of nonlinear normal modes of symmetric Hamiltonian systems, Nonlinearity, 3, 695730, (1990).

[14] Moser, J., Periodic orbits near equilibria and theorem by Alan Weinstein, Commun. Pure Appl. Math., 29, 727-747, (1976).

[15] Poènaru, V., Singularités $C^{\infty}$ en Présence de Symétrie, Lecture notes in mathematics, 510, (1976).

[16] Takens, F., Singularities of vector fields, Publ. Math. IHES, 43, 47-100, (1974).

[17] Vanderbauwhede, A., Local bifurcation and symmetry, Res. Notes in Math., 75, Pitman, Boston, (1982).

[18] Van der Meer, J. -C., The Hamiltonian Hopf bifurcation, Lec. Notes in Math., 1160, Springer, (1985).

[19] Van der Meer, J. -C., Sanders, J. and Vanderbauwhede, A. Hamiltonian structure of the reversible nonsemisimple 1: 1 resonance. In: Dynamics, bifurcation and Symmetry (Cargèse, 1993), 221-240, NATO Adv. Sci. Inst. Ser. C. Math. Phys. Sci., 437, Kluver Acad. Publ. Dordrecht, (1994).

[20] Weinstein, A., Normal modes for nonlinear Hamiltonian systems, Invent. Math., 20, 47-57, (1973). 